\title{
Increased Plasma Levels of 8-Hydroxy-2'-deoxyguanosine (8-OHdG) in Patients with Pseudoexfoliation Glaucoma
}

\author{
Altaf A. Kondkar $\mathbb{D}^{1,2}$ Tahira Sultan, ${ }^{1}$ Taif A. Azad ${ }^{1},{ }^{1}$ Lubna Tabussum, ${ }^{3}$ \\ Essam A. Osman, ${ }^{1}$ and Saleh A. Al-Obeidan $\mathbb{B}^{1,2}$ \\ ${ }^{1}$ Department of Ophthalmology, College of Medicine, King Saud University, Riyadh, Saudi Arabia \\ ${ }^{2}$ Glaucoma Research Chair in Ophthalmology, College of Medicine, King Saud University, Riyadh, Saudi Arabia \\ ${ }^{3}$ Ambulatory Care, King Saud University, Riyadh, Saudi Arabia
}

Correspondence should be addressed to Altaf A. Kondkar; akondkar@gmail.com

Received 4 January 2019; Accepted 19 May 2019; Published 1 July 2019

Academic Editor: Vicente Zanon-Moreno

Copyright (C) 2019 Altaf A. Kondkar et al. This is an open access article distributed under the Creative Commons Attribution License, which permits unrestricted use, distribution, and reproduction in any medium, provided the original work is properly cited.

Purpose. To investigate systemic oxidative stress-induced DNA damage in patients with pseudoexfoliation glaucoma (PXG), we estimated plasma levels of 8-hydroxy-2'-deoxyguanosine (8-OHdG) as a marker for oxidative DNA damage in comparison to controls. In addition, we also examined a combined effect of lysyl oxidase-like 1 (LOXL1) polymorphism status and 8-OHdG levels on PXG risk. Materials and Methods. A retrospective case-control study was performed to estimate plasma levels of 8-OHdG in 41 PXG patients and 45 nonglaucomatous controls using the enzyme-linked immunosorbent assay (ELISA). The assay was performed in duplicate on an automated ELISA analyzer. Two common polymorphisms (rs1048661 and rs3835942) in LOXL1 gene were genotyped by Sanger sequencing. Results. The mean and median levels of 8-OHdG were significantly increased in the PXG cases $(p=0.032)$ and male subjects $(p=0.041)$. Subjects with levels greater than the third quartile $(75 \%$ percentile $)$ exhibited a significant increased risk of PXG (odds ratio $=4.06,95 \%$ confidence interval $(\mathrm{CI}=1.11-14.80, p=0.029)$ ). Within- and betweengroup comparisons showed that the mean levels were higher in individuals carrying the LOXL1 risk variant (G/G), but not statistically significant. In logistic regression analysis, both 8-OHdG $(p=0.044)$ and $\mathrm{rs} 3835942(p=0.012)$ showed a statistically significant effect on the PXG outcome. However, the effect was lost when age, sex, and rs1048661 were included. A significant positive correlation was observed between 8 -OHdG levels and intraocular pressure $(R=0.284, p=0.008)$ and cup/disc ratio $(R=0.233, p=0.031)$. Furthermore, in receiver operating characteristic analysis, the area under the curve was statistically significant $(p=0.032)$ with a value of $0.635(95 \% \mathrm{CI}=0.518-0.751)$. Conclusion. The study demonstrates an association of increased plasma levels of 8-OHdG in patients with PXG, supporting the role of oxidative stress, and increased oxidative DNA damage in PXG development.

\section{Introduction}

Glaucoma is one of the leading causes of irreversible blindness worldwide [1], including in Saudi Arabia [2]. As a result of blocked aqueous humor outflow at the trabecular meshwork (TM), elevated intraocular pressure (IOP) is considered as a primary risk factor that triggers the death of retinal ganglion cells (RGCs) and progressive loss of RGC axons, causing glaucomatous optic neuropathy [3]. Pseudoexfoliation glaucoma (PXG) is often characterized by accumulation of abnormal fibrillar extraceullular materials in the anterior segment of the eye, primarily along the pupillary border, which is considered as a pathologic hallmark of the disease [4]. In comparison with more common primary open-angle glaucoma (POAG), patients with PXG often show a more aggressive clinical course with higher IOP, larger cupping, severe visual field defects, and worse prognosis with rapid progression of the disease $[3,4]$. There is a lack of systematic epidemiological studies in the literature to know the exact prevalence of PXG in Saudi Arabia. However, it has been observed that from approximately 600 new glaucoma cases registered at our glaucoma unit of King Abdulaziz University Hospital in Riyadh, less than 10\% of these cases are diagnosed with PXG. 
PXG represents a complex and multifactorial adult-onset disease. The disease involves both genetic and environmental factors that contribute to the etiological pathophysiology of the disease [5]. Lysyl oxidase-like 1 (LOXL1) is a strong candidate gene reported to contribute to the risk of PXG development in multiple ethnic groups, including Saudi Arabians. LOXL1 is involved with extracellular matrix formation and stability $[5,6]$. There are strong lines of evidence to suggest a role of oxidative stress mechanism(s) in pathogenesis of PXG [7-10]. Oxidative stress is generally induced through the formation of reactive oxygen species (ROS) such as superoxide, peroxide, and hydroxyl radicals that can initiate and propagate free radicals [7]. The accumulation of ROS levels in the cells induces oxidative damage in macromolecules like lipids, proteins, RNA, DNA, and mitochondria, resulting in their cellular dysfunction and/or apoptosis [7]. It has been demonstrated that oxidative stress causes an increase in the IOP by initiating TM degeneration, thereby hindering the aqueous outflow pathway [11]. Mutations, haplogroups, and decreased respiratory activities in the mitochondria have also been associated with various types of glaucoma $[12,13]$. Besides, a polymorphism in glutathione S-transferase (GST) gene, an enzyme involved in detoxifying peroxidized lipids and various harmful toxins, has also been associated with glaucoma [14]. Reduced GST activity may interfere with detoxification of oxidative metabolites and aggravate the damaging effects of oxidative stress on the optic nerve [14]. The total antioxidant status (TAS) of biological samples is an important indicator of oxidative stress and a useful tool to predict oxidative status [9]. Our previous study has shown that plasma TAS levels were significantly low in PXG patients as compared to controls, supporting the role of oxidative stress in the pathogenesis of PXG [9]. Besides, we also reported a combined effect of the LOXL1 alleles and the decreased TAS that may contribute to the overall risk of PXG. In humans, in vivo experiments have demonstrated that oxidative DNA damage is significantly more abundant in the TM cells of glaucoma patients. In addition, both increased IOP and visual field damage were significantly related to the amount of oxidative DNA damage affecting TM cells $[11,15]$. Oxidative stress/ROS can induce breaks or base modifications in the DNA resulting in the release of DNA oxidation products, including 8-hydroxy-2' -deoxyguanosine (8-OHdG) [16]. $8-\mathrm{OHdG}$ is one of the multiple products of DNA oxidation that can be easily quantified and is commonly used as a biomarker to assess oxidative DNA damage [17].

The aim of this study was to investigate systemic oxidative stress-induced DNA damage in patients with PXG. We estimated plasma levels of $8-\mathrm{OHdG}$ as a marker for oxidative DNA damage and compared it with nonglaucomatous healthy controls. In addition, we also investigated the combined effect of LOXL1 polymorphism status and 8-OHdG level on the risk of PXG.

\section{Materials and Methods}

2.1. Study Population. The study adhered to the tenets of the Declaration of Helsinki and was approved by the institutional review board and research ethics committee (approval number \# 08-657). Following written informed consent, participants of Saudi origin with established clinical diagnosis of PXG $(n=41)$ and ethnically matched healthy controls $(n=45)$ were recruited for the study at King Abdulaziz University Hospital in Riyadh, Saudi Arabia. The inclusion-exclusion criteria of patients and controls have been described previously [10].

2.2. Plasma and DNA Preparation. Plasma samples were obtained from EDTA blood following centrifugation at $5500 \times \mathrm{g}$ for $5 \mathrm{~min}$. DNA was extracted from the buffy layer using the illustra blood genomicPrep Mini Spin Kit (GE Healthcare, Buckinghamshire, UK). Storage was at $-80^{\circ} \mathrm{C}$ until use.

2.3. Estimation of Plasma Levels of 8-Hydroxy $2^{\prime}$ Deoxyguanosine. The estimation of $8-\mathrm{OHdG}$ levels was performed using a commercial kit (Trevigen, Gaithersburg, MD, USA) based on a competitive sandwich enzymelinked immunosorbent assay (ELISA). The assay was performed in duplicate on a ChemWell-T automated ELISA analyzer (Awareness Technology Inc., FL, USA), as per the manufacturer's instructions. The 8-OHdG levels were established utilizing the standard curve and expressed in $\mathrm{ng} / \mathrm{mL}$.

2.4. LOXL1 Sanger Sequencing. DNA samples were sequenced for the two common LOXL1 gene polymorphisms (rs1048661 and rs3825942) using primers and amplification conditions as described elsewhere [9].

2.5. Statistical Analysis. Data are presented as mean $\pm \mathrm{SD}$ and median for continuous variables and as counts and percentages for categorical variables. Normality testing for 8-OHdG levels was done using the Kolmogorov-Smirnov test. Mean differences between groups were tested by Student's $t$-test. The Mann-Whitney $U$ test was used to compare median values between the patients and controls. The categorical variables were tested by the chi-square test and Fisher's exact test where applicable. The correlation testing was done using Pearson's method. A binary logistic regression analysis was performed to estimate the impact and effect of mean 8-OHdG levels and other risk factors on disease outcome. A receiver operating characteristic (ROC) curve was generated, and the area under the curve (AUC) value was analyzed by the Mann-Whitney test. Odds ratio (OR) was calculated, and a confidence interval (CI) was set to $95 \%$. All statistical tests were two-sided, and a $p$ value less than 0.05 was considered statistically significant. Statistical analysis was performed with SPSS version 19.0 (IBM Corp., Armonk, New York, USA) and StatView software version 5.0 (SAS Institute, Cary, NC).

\section{Results}

3.1. Study Population and $8-O H d G$ Levels. As shown in Table 1, there was no significant difference between PXG 
TABLE 1: Demographic characteristics and 8-hydroxydeoxyguanosine (8-OHdG) levels between pseudoexfoliation glaucoma cases and controls.

\begin{tabular}{lccc}
\hline Variables & $\begin{array}{c}\text { Cases } \\
(n=41)\end{array}$ & $\begin{array}{c}\text { Controls } \\
(n=45)\end{array}$ & $\begin{array}{c}p \\
\text { value }\end{array}$ \\
\hline $\begin{array}{l}\text { Age in years, mean (SD) } \\
\text { Male/female, } n\end{array}$ & $\begin{array}{c}62.27(6.31) \\
\text { Systemic diseases, } n(\%)\end{array}$ & $\begin{array}{c}59.91(8.33) \\
0.146^{\mathrm{a}}\end{array}$ \\
$\begin{array}{l}\text { Diabetes mellitus } \\
\text { Hypertension }\end{array}$ & $6(14.6)$ & $6(13.3)$ & $0.861^{\mathrm{b}}$ \\
Coronary artery disease & $4(9.7)$ & $5(11.1)$ & $0.605^{\mathrm{b}}$ \\
Hypercholesterolemia & $2(4.8)$ & $2(4.4)$ & $0.924^{\mathrm{b}}$ \\
Family history of glaucoma & $3(7.3)$ & $2(4.4)$ & $0.569^{\mathrm{b}}$ \\
Smokers & $5(12.2)$ & $1(2.2)$ & $0.069^{\mathrm{b}}$ \\
\hline
\end{tabular}

8-OHdG concentration,

$n g / m L$

$\begin{array}{llll}\text { Mean (SD) } \quad 30.48(31.52) & 16.95(10.66) & 0.008^{\mathrm{a}}\end{array}$

\begin{tabular}{llll} 
Median & 18.11 & 13.78 & $0.032^{\mathrm{c}}$ \\
\hline
\end{tabular}

By gender

Males, mean (SD)

Median

Females, mean (SD)

Median

$\begin{array}{ccc}29.53(26.94) & 17.02(11.02) & 0.023^{\mathrm{a}} \\ 18.17 & 13.12 & 0.041^{\mathrm{c}} \\ 32.12(39.22) & 16.80(10.29) & 0.154^{\mathrm{a}} \\ 17.68 & 17.97 & 0.442^{\mathrm{c}}\end{array}$

Notes. ${ }^{\mathrm{a}}$ Independent sample $t$-test (two-tailed); ${ }^{\mathrm{b}}$ chi-square test; ${ }^{\mathrm{c}}$ MannWhitney $U$ test.

cases and controls for age, gender, systemic disease status, smoking, and family history of glaucoma. Normality testing for 8-OHdG levels showed a skewed distribution $(p<0.001)$. Both mean and median 8-OHdG levels were significantly elevated in the PXG cases and male subjects as compared to the controls (Table 1). Figures $1(\mathrm{a})$ and $1(\mathrm{~b})$ show the box-plot representation of 8-OHdG levels according to disease status and gender distribution, respectively.

3.2. Levels of 8-OHdG and Risk of PXG. To assess the risk of PXG associated with increasing levels of 8-OHdG, the 8OHdG concentrations were dichotomized (uncategorized as cases and controls) at the $50^{\text {th }}$ percentile (or median value) and by quartiles (Table 2). The overall median cutoff of the 8OHdG level was $17.68 \mathrm{ng} / \mathrm{mL}$. Although cases showed an increased risk of disease at this level $(\mathrm{OR}=1.76,95 \%$ $\mathrm{CI}=0.75-4.15)$, the difference was nonsignificant $(p=0.190)$. Similarly, using quartile distribution, two cutoff levels identified were $10.08 \mathrm{ng} / \mathrm{mL}$ (the first quartile or the $25^{\text {th }}$ percentile) and $27.72 \mathrm{ng} / \mathrm{mL}$ (the third quartile or the $75^{\text {th }}$ percentile). Using these two cutoff values, subjects were then categorized into three groups: less than the first quartile, the interquartile, and greater than the third quartile (Table 2). Overall, there was no significant additive effect of increasing levels of 8 -OHdG and PXG outcome $\left(\chi^{2}=4.87, \mathrm{df}=2\right.$; $p=0.0875)$. In addition, compared to 8-OHdG levels less than the first quartile $(<10.08 \mathrm{ng} / \mathrm{mL})$, subjects with interquartile levels showed a nonsignificant increased risk of disease $(\mathrm{OR}=2.50,95 \% \mathrm{CI}=0.81-7.63, p=0.103)$, whereas subjects with levels greater than the third quartile $\left(75^{\text {th }}\right.$ percentile) exhibited a significant increased risk of PXG $(\mathrm{OR}=4.06,95 \% \mathrm{CI}=1.11-14.80, p=0.029)$.
3.3. 8-OHdG Levels and LOXL1 Polymorphisms. We further investigated the genotype effect of polymorphisms rs1048661 (g.5758 G>T) and rs3825942 (g.5758 G>A) in the LOXL1 gene on 8-OHdG levels in PXG cases and controls. Overall, as shown in Table 3, there was no significant difference between 8-OHdG levels and different genotypes for both LOXL1 polymorphisms (Figures 1(c) and 1(d)).

For rs1048661, G/G was the most common genotype, followed by G/T and T/T. No T/T homozygosity was observed in the patient group as compared to only one in the controls. The mean 8-OHdG levels were observed to be highest in the G/G genotypes as compared to G/T, T/T, or G/ $\mathrm{T}+\mathrm{T} / \mathrm{T}$ groups. However, these levels did not vary significantly both within and between the study group comparisons.

Similarly, for rs383592, G/G was the most prevalent genotype, followed by G/A and A/A genotypes, with the latter being absent in PXG patients. G/G was the most common genotype exhibiting highest levels of 8-OHdG in comparison to G/A, A/A, or G/A + A/A groups. The levels did not vary significantly between cases and controls for $\mathrm{G} / \mathrm{G}$ genotypes $(p=0.369)$. In addition, a within-group comparison in controls also did not show any significant genotype effect on 8 -OHdG for G/A $(p=0.061)$, A/A, $(p=0.818)$ and $\mathrm{G} / \mathrm{A}+\mathrm{A} / \mathrm{A}(p=0.118)$ as compared to $\mathrm{G} / \mathrm{G}$ genotype.

3.4. 8-OHdG Levels and Other Risk Factors. With a view to examine the effect of age, sex, LOXL1 polymorphisms, and 8OHdG levels in patients with PXG, a binary logistic regression analysis was performed using diseased/nondiseased as a dependent variable (outcome). The analysis showed that both 8-OHdG $(p=0.044)$ and $\mathrm{rs} 3835942(p=0.012)$ have statistically significant effect on the disease outcome. However, in a combined analysis with age, sex, 8-OHdG, rs1048661, and rs3835942, none of these risk factors showed any significant impact on PXG (Table 4).

3.5. Correlation between 8-OHdG and Other Glaucoma Indices in PXG Patients. A significant positive correlation was observed between 8-OHdG and IOP $(R=0.284, p=0.008)$ and cup/disc ratio $(R=0.233, p=0.031)$, as opposed to none with age $(R=0.154, p=0.157)$ and number of antiglaucoma medication $(R=-0.035, p=0.829)$.

3.6. ROC Curve and 8-OHdG. ROC curve analysis of 8OHdG levels in PXG patients and controls revealed an AUC of $0.635(95 \% \mathrm{CI}=0.518-0.751)$ that was statistically significant ( $p=0.032$ ), indicating that the plasma levels of 8 OHdG could satisfactorily discriminate between PXG patients and controls.

\section{Discussion}

Increased ROS, oxidative damage, and imbalance between prooxidant status and antioxidant status are critical factors which significantly contribute to glaucomatous 


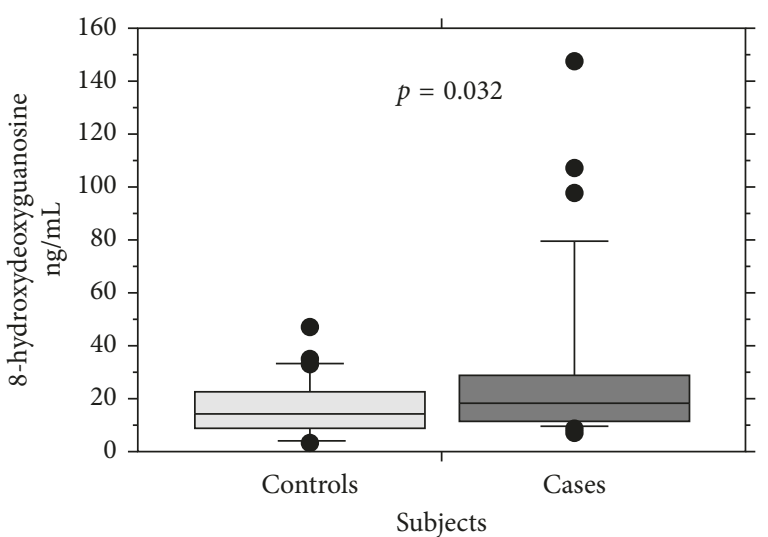

(a)

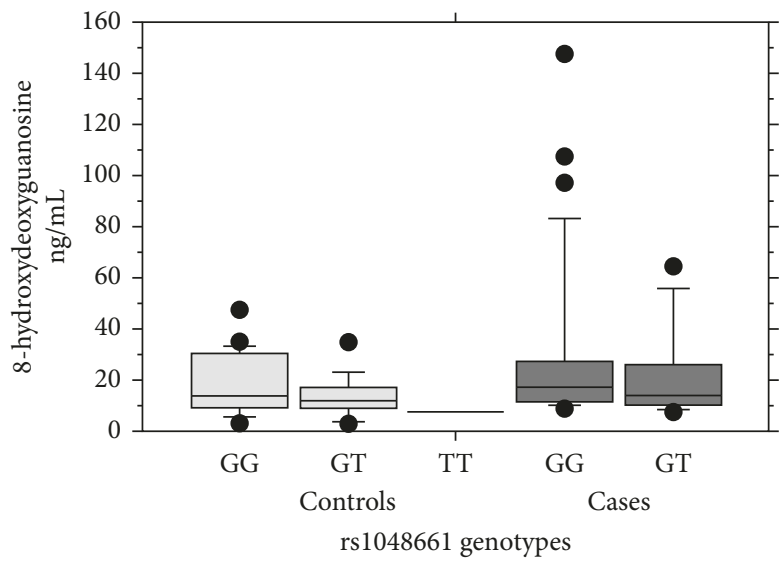

(c)

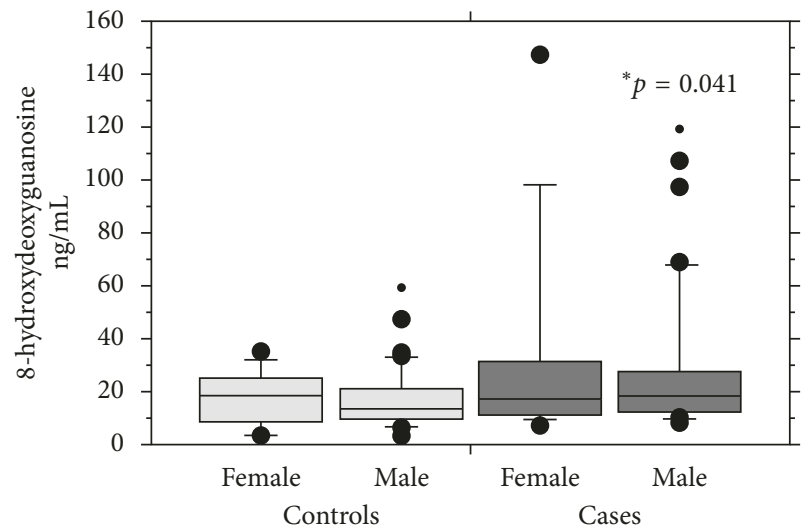

(b)

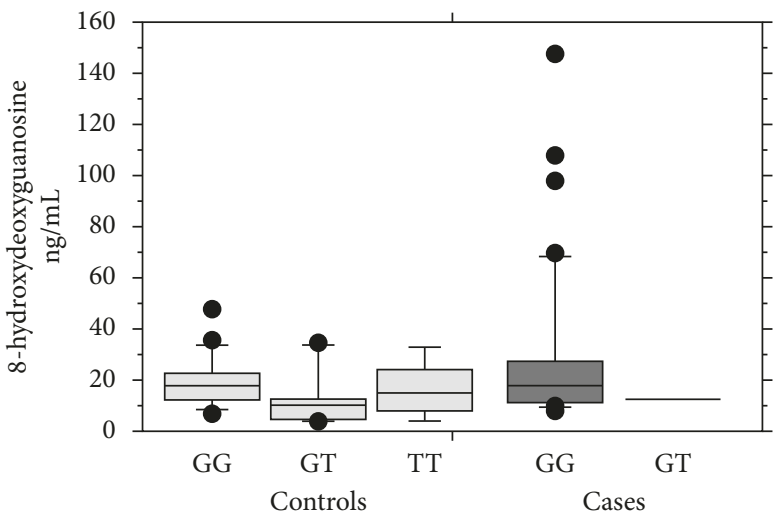

rs3825942 genotypes

(d)

Figure 1: Box plot showing distribution of 8-hydroxydeoxyguanosine levels between cases and controls of (a) pseudoexfoliation glaucoma (PXG), (b) males and females, (c) LOXL1 rs1048661 genotypes, and (d) LOXL1 rs3825942 genotypes. Differences were tested by the Mann-Whitney $U$ test both within and between the study groups. Significance was observed only between cases and controls and between control males vs. PXG males as indicated in (a) and (b), respectively. No other significant distribution was observed.

TABLE 2: Evaluation of risk of pseudoexfoliation glaucoma according to median and quartile levels of 8-hydroxydeoxyguanosine (8-OHdG).

\begin{tabular}{|c|c|c|c|c|c|}
\hline 8-OHdG ng/mL & No. of cases (\%) & No. of controls (\%) & Odds ratio & 95\% confidence interval & $p$ value $^{\mathrm{a}}$ \\
\hline \multicolumn{6}{|l|}{ By median } \\
\hline$<17.68$ & $17(41.4)$ & $25(55.5)$ & Reference & - & - \\
\hline$\geq 17.68$ & $24(58.5)$ & $20(44.5)$ & 1.76 & $0.75-4.15$ & 0.190 \\
\hline \multicolumn{6}{|l|}{ By quartiles ${ }^{\dagger}$} \\
\hline$<10.08$ & $6(14.6)$ & $15(33.3)$ & Reference & - & - \\
\hline $10.08-27.72$ & $22(53.6)$ & $22(48.8)$ & 2.50 & $0.81-7.63$ & 0.103 \\
\hline$>27.72$ & $13(31.7)$ & $8(17.8)$ & 4.06 & $1.11-14.80$ & 0.029 \\
\hline
\end{tabular}

Note. ${ }^{\text {a }}$ Chi-square test; ${ }^{\dagger}$ first quartile $\left(<25^{\text {th }}\right.$ percentile); interquartile $\left(25^{\text {th }}-75^{\text {th }}\right.$ percentile); third quartile $\left(>75^{\text {th }}\right.$ percentile).

neurodegeneration [7, 18]. This study reports increased levels of systemic 8-OHdG, a marker of oxidative stressinduced DNA damage, in patients with PXG.

DNA damage can be inflicted by both extrinsic and intrinsic agents such as ionizing radiations, ultraviolet light, toxic chemicals/metal ions, and ROS generated as a consequence of normal cellular metabolism of oxygen [19]. The endogenous processes that may plausibly contribute to an ongoing DNA damage in vivo include oxidative methylation, depurination, and deamination [16, 19]. ROSinduced damages include base and sugar lesions, protein, and DNA cross-links, and single-strand/double-strand breaks. Moreover, the base guanine is most susceptible to oxidative modifications because of its least redox potential $[16,19]$. The most common byproduct formed due to oxidative modification of guanine by hydroxyl radical is 8hydroxyguanine (8-OH-Gua) and its $2^{\prime}$-deoxynucleoside equivalent, $8-\mathrm{OHdG}$, which is proposed to be an excellent marker for oxidative damage to DNA [20]. A number of studies have provided strong evidence for association between increased levels of 8-OHdG and glaucomatous optic neuropathy $[11,15,21]$. 
TABLE 3: Levels of 8-hydroxydeoxyguanosine (8-OHdG) according to LOXL1 rs1048661 and rs3835942 polymorphisms in pseudoexfoliation glaucoma cases and controls.

\begin{tabular}{lccc}
\hline LOXL1 genotypes & \multicolumn{2}{c}{$\begin{array}{c}\text { 8-OHdG } \mathrm{ng} / \mathrm{mL} \text {, mean }(\mathrm{SD}) \\
\text { Controls* }\end{array}$} & Cases* \\
\hline rs1048661 & & & \\
$\mathrm{G} / \mathrm{G}$ & $18.55(12.04)$ & $30.16(17.90)$ & 0.236 \\
$\mathrm{G} / \mathrm{T}$ & $13.71(8.16)$ & $21.99(19.31)$ & 0.617 \\
$\mathrm{~T} / \mathrm{T}$ & 7.84 & - & - \\
$\mathrm{G} / \mathrm{T}+\mathrm{T} / \mathrm{T}$ & $13.26(7.98)$ & $21.99(19.31)$ & 0.469 \\
\hline$r s 3825942$ & & & \\
$\mathrm{G} / \mathrm{G}$ & $18.53(10.68)$ & $28.89(30.97)$ & 0.369 \\
$\mathrm{G} / \mathrm{A}$ & $12.93(11.46)$ & 11.83 & - \\
$\mathrm{A} / \mathrm{A}$ & $16.13(11.33)$ & - & - \\
$\mathrm{G} / \mathrm{A}+\mathrm{A} / \mathrm{A}$ & $14.00(11.11)$ & 11.83 & - \\
\hline
\end{tabular}

Note. ${ }^{*} p$ values tested within groups using $\mathrm{G} / \mathrm{G}$ as reference by the Mann-Whitney $U$ test were also nonsignificant $(p>0.05)$.

TABLE 4: Binary logistic regression analysis of risk variables on disease outcome.

\begin{tabular}{lcc}
\hline Risk variables & Odds ratio $(95 \%$ confidence interval) & $p$ value \\
\hline Age & $1.035(0.964-1.112)$ & 0.345 \\
Sex $^{\mathrm{a}}$ & $1.150(0.352-3.762)$ & 0.817 \\
8-OHdG $^{\mathrm{b}}$ & $1.035(0.985-1.053)$ & 0.292 \\
rs1048661 $^{\mathrm{c}}$ & - & 0.599 \\
rs3835942 & - & 0.073 \\
\hline
\end{tabular}

Note. ${ }^{\mathrm{a}}$ Females as reference; ${ }^{\mathrm{b}}$ 8-OHdG, 8-hydroxydeoxyguanosine; ${ }^{\mathrm{c}} \mathrm{G} / \mathrm{G}$ genotypes as reference.

Initial studies on human TM specimens obtained during filtration surgery have demonstrated presence of high levels of 8 -OHdG in patients with glaucoma $[11,15]$. In a study by Sorkhabi et al. that included 15 POAG and 13 PXG patients, both aqueous humor (AH) and serum 8-OHdG levels were high in patients as compared to controls [21]. Similarly, high levels of serum 8-OHdG have been reported in patients with primary angle closure glaucoma as compared to normal subjects [22]. Yuki and Tsubota reported an increased urinary $8-\mathrm{OHdG} /$ creatinine level to be associated with glaucomatous visual field progression in subjects with normal tension glaucoma [23]. Likewise, in another recent study by Mohanty et al., both plasma and AH 8-OHdG levels were significantly higher in POAG patients in comparison to cataract controls [24]. This effect was attributed to reduced expression of DNA repair enzymes of the base excision repair pathway [24]. In addition, the study also reported a strong positive correlation between systemic (plasma) 8OHdG levels and AH 8-OHdG levels, suggesting that systemic 8-OHdG levels could be predictive of local 8-OHdG levels in the eye [24].

The exact role of 8-OHdG and/or oxidative stress leading to the development and progression of glaucomatous optic neuropathy is still speculative. $8-\mathrm{OHdG}$ is among the best characterized oxidative lesions, and it can give rise to $\mathrm{C}: \mathrm{G}$ to $\mathrm{A}: \mathrm{T}$ transversion mutations [25]. Some lesions in DNA are subjected to cellular repair by in vivo DNA repair mechanisms that cleave off the damaged DNA [25]. However, failure to repair these damages can have serious biological implications and may lead to carcinogenesis or development of neurodegenerative disorders [20, 24, 26, 27]. 8-OHdG can also significantly induce telomere shortening that may contribute to physiological and pathological conditions in vivo $[25,28]$. Furthermore, it has been suggested that 8OHdG may have an epigenetic-like regulatory role in cells undergoing oxidative stress in regulation of gene transcription [25]. Moreover, the abnormal effects of oxidative stress and ROS in glaucoma pathogenesis via increased IOP and/or hypoxia, TM degeneration, glial cell damage, autophagy, mtDNA damage, nuclear-kappa $B$ activation, peroxynitrite stress, and ocular hemodynamics stimulating apoptosis and inflammatory pathways to promote RGC death and optic nerve damage have been well documented [7]. Increased levels of 8-OHdG observed in our study may plausibly contribute to PXG pathogenesis by similar mechanism(s).

A strong association of two missense variants, rs1048661 and rs3825942, in the LOXL1 gene with PXG has been consistently replicated in multiple ethnic groups including Saudi Arabians [5, 6]. A complete understanding of this genetic association to PXG pathology is still not known; however, a number of mechanisms have been postulated. These include its effect on elastin formation, altered LOXL1 expression, splicing-effect, or possibility of their association/ linkage with an actual unidentified functional allele [5]. We investigated the genotype effects of these two common variants on $8-\mathrm{OHdG}$ and their combined effect on the risk of PXG. The genotypes were not found to influence the 8OHdG levels, although the levels were high in the risk (G/G) variants. Both 8-OHdG and rs3835942 were found to be significant risk factors for PXG. However, this significance was lost in a combined analysis that also included age, sex, and rs1048861 genotypes. A lack of sufficient numbers in each genotype group could be a likely explanation to this loss and absence of any significant effect.

An increased IOP and visual field damage have been significantly correlated to the amount of oxidative DNA damage in the TM cells $[11,15]$. Similarly, the IOP and cup/ disc ratio also showed a significant positive correlation with 8-OHdG levels in our study, suggestive of its possible utility as a marker of disease severity. Besides, a number of reports have supported an existence of inverse relation between antioxidant defense mechanism(s) and oxidative stress in the pathophysiology of glaucoma $[9,18,21,29]$. Our group has also previously reported significantly reduced plasma total antioxidant status (TAS) in PXG patients as compared to controls [9]. Interestingly, TAS levels were available for the PXG patients (not for controls; data not shown) included in this study that showed a significant negative correlation between 8 -OHdG and TAS in PXG patients $(R=-0.365$, $p=0.022$ ), supporting the plausible role of increased oxidative stress and decreased antioxidant defense mechanisms in PXG.

The findings of this study require cautious interpretation because of its certain limitations. First, we have to acknowledge the fact that the systemic increase in 8-OHdG may not exactly reflect the situation in the anterior chamber of the eye, where cells/tissues are constantly exposed to a higher amount of free radical insults and thus are more directly 
involved in the development and progression of glaucoma through the oxidative stress mechanisms. This would require an additional validation in AH samples. Second, the study is purely descriptive in nature and does not provide any temporal or mechanistic evidence or suggest any causal implications of elevated 8-OHdG in PXG. Finally, a relatively small number of samples have been examined in this study. A replication in a much larger cohort would certainly strengthen the observations of this study. Nonetheless, considering a mean difference (effect size) of $13 \mathrm{ng} / \mathrm{mL}$ in 8OHdG concentration as observed between PXG cases and controls, with an average standard deviation of 20 and type I error of 0.05 (two-sided), the study exhibits a power of $>80 \%$.

\section{Conclusion}

To conclude, the study demonstrates an increased level of systemic 8-OHdG in patients with PXG, supporting an association of this marker with PXG, and the plausible role of oxidative stress and increased oxidative DNA damage in PXG etiology.

\section{Data Availability}

The data supporting the conclusions of this article are all presented within the article.

\section{Ethical Approval}

The study adhered to the tenets of the Declaration of Helsinki and had received approval from the Institutional Review Board and Research Ethics Committee of College of Medicine, King Saud University, Riyadh, Saudi Arabia.

\section{Consent}

Written informed consent was obtained from all participants prior to their inclusion in this study.

\section{Conflicts of Interest}

The authors report no conflicts of interest. The funder had no role in the design of the study, in collection, analysis, and interpretation of data, and in writing the manuscript. The authors alone are responsible for the content and writing of the paper.

\section{Acknowledgments}

The authors would like to thank the Vice Deanship of Scientific Research Chair and Gluacoma Research Chair in Ophthalmology at the King Saud University through whom this work was supported.

\section{References}

[1] A. Foster and S. Resnikoff, "The impact of vision 2020 on global blindness," Eye, vol. 19, no. 10, pp. 1133-1135, 2005.

[2] S. A. Al Obeidan, A. Dewedar, E. A. Osman, and A. Mousa, "The profile of glaucoma in a tertiary ophthalmic university center in Riyadh, Saudi Arabia," Saudi Journal of Ophthalmology, vol. 25, no. 4, pp. 373-379, 2011.

[3] R. Ritch, "Ocular and systemic manifestations of exfoliation syndrome," Journal of Glaucoma, vol. 23, no. 8, pp. S1-S8, 2014.

[4] R. Ritch, U. Schlotzer-Schrehardt, and A. G. Konstas, "Why is glaucoma associated with exfoliation syndrome?," Progress in Retinal and Eye Research, vol. 22, no. 3, pp. 253-275, 2003.

[5] B. T. Whigham and R. R. Allingham, "Review: the role of LOXL1 in exfoliation syndrome/glaucoma," Saudi Journal of Ophthalmology, vol. 25, no. 4, pp. 347-352, 2011.

[6] K. K. Abu-Amero, E. A. Osman, A. S. Dewedar, S. Schmidt, R. R. Allingham, and S. A. Al-Obeidan, "Analysis of LOXL1 polymorphisms in a Saudi Arabian population with pseudoexfoliation glaucoma," Molecular Vision, vol. 16, pp. 2805-2810, 2010.

[7] M. Nita and A. Grzybowski, "The role of the reactive oxygen species and oxidative stress in the pathomechanism of the agerelated ocular diseases and other pathologies of the anterior and posterior eye segments in adults," Oxidative Medicine and Cellular Longevity, vol. 2016, Article ID 3164734, 23 pages, 2016.

[8] M. Zenkel, P. Lewczuk, A. Jünemann, F. E. Kruse, G. O. H. Naumann, and U. Schlötzer-Schrehardt, "Proinflammatory cytokines are involved in the initiation of the abnormal matrix process in pseudoexfoliation syndrome/ glaucoma," American Journal of Pathology, vol. 176, no. 6, pp. 2868-2879, 2010.

[9] K. K. Abu-Amero, A. A. Kondkar, A. Mousa, E. A. Osman, and S. A. Al-Obeidan, "Decreased total antioxidants status in the plasma of patients with pseudoexfoliation glaucoma," Molecular Vision, vol. 17, pp. 2769-2775, 2011.

[10] A. Kondkar, T. A. Azad, F. Almobarak, H. Kalantan, S. AlObeidan, and K. Abu-Amero, "Elevated levels of plasma tumor necrosis factor alpha in patients with pseudoexfoliation glaucoma," Clinical Ophthalmology, vol. 12, pp. 153-159, 2018.

[11] A. Izzotti, C. Cartiglia, S. De Flora, and S. Saccà, "Methodology for evaluating oxidative DNA damage and metabolic genotypes in human trabecular meshwork," Toxicology Mechanisms and Methods, vol. 13, no. 3, pp. 161-168, 2003.

[12] K. K. Abu-Amero, T. M. Bosley, and J. Morales, "Analysis of nuclear and mitochondrial genes in patients with pseudoexfoliation glaucoma," Molecular Vision, vol. 14, pp. 29-36, 2008.

[13] K. K. Abu-Amero, V. M. Cabrera, J. M. Larruga, E. A. Osman, A. M. Gonzalez, and S. A. Al-Obeidan, "Eurasian and SubSaharan African mitochondrial DNA haplogroup influences pseudoexfoliation glaucoma development in Saudi patients," Molecular Vision, vol. 17, pp. 543-547, 2011.

[14] K. K. Abu-Amero, J. Morales, G. H. Mohamed, M. N. Osman, and T. M. Bosley, "Glutathione S-transferase M1 and T1 polymorphisms in Arab glaucoma patients," Molecular Vision, vol. 14, pp. 425-430, 2008.

[15] S. C. Saccà, A. Pascotto, P. Camicione, P. Capris, and A. Izzotti, "Oxidative DNA damage in the human trabecular meshwork," Archives of Ophthalmology, vol. 123, no. 4, pp. 458-463, 2005.

[16] T. Hemnani and M. S. Parihar, "Reactive oxygen species and oxidative DNA damage," Indian Journal of Physiology and Pharmacology, vol. 42, no. 4, pp. 440-452, 1998.

[17] I. Dalle-Donne, R. Rossi, R. Colombo, D. Giustarini, and A. Milzani, "Biomarkers of oxidative damage in human disease," Clinical Chemistry, vol. 52, no. 4, pp. 601-623, 2006. 
[18] A. Izzotti, A. Bagnis, and S. Sacca, "The role of oxidative stress in glaucoma," Mutation Research/Reviews in Mutation Research, vol. 612, no. 2, pp. 105-114, 2006.

[19] J. Cadet and J. R. Wagner, "DNA base damage by reactive oxygen species, oxidizing agents, and UV radiation," Cold Spring Harbor Perspectives in Biology, vol. 5, no. 2, 2013.

[20] A. Valavanidis, T. Vlachogianni, and C. Fiotakis, "8-hydroxy$2^{\prime}$-deoxyguanosine (8-OHdG): a critical biomarker of oxidative stress and carcinogenesis," Journal of Environmental Science and Health, Part C, vol. 27, no. 2, pp. 120-139, 2009.

[21] R. Sorkhabi, A. Ghorbanihaghjo, A. Javadzadeh, N. Rashtchizadeh, and M. Moharrery, "Oxidative DNA damage and total antioxidant status in glaucoma patients," Molecular Vision, vol. 17, pp. 41-46, 2011.

[22] D. Chang, Q. Sha, X. Zhang et al., "The evaluation of the oxidative stress parameters in patients with primary angleclosure glaucoma," PLoS One, vol. 6, no. 11, Article ID e27218, 2011.

[23] K. Yuki and K. Tsubota, "Increased urinary 8-Hydroxy-2'deoxyguanosine (8-OHdG)/creatinine level is associated with the progression of normal-tension glaucoma," Current Eye Research, vol. 38, no. 9, pp. 983-988, 2013.

[24] K. Mohanty, R. Dada, and T. Dada, "Oxidative DNA damage and reduced expression of DNA repair genes: role in primary open angle glaucoma (POAG)," Ophthalmic Genetics, vol. 38, no. 5, pp. 446-450, 2017.

[25] E. Markkanen, "Not breathing is not an option: how to deal with oxidative DNA damage," DNA Repair, vol. 59, pp. 82105, 2017.

[26] M. S. Lillenes, A. Rabano, M. Stoen et al., "Altered DNA base excision repair profile in brain tissue and blood in Alzheimer's disease," Molecular Brain, vol. 9, no. 1, p. 61, 2016.

[27] N. Kubo, M. Morita, Y. Nakashima et al., "Oxidative DNA damage in human esophageal cancer: clinicopathological analysis of 8-hydroxydeoxyguanosine and its repair enzyme," Diseases of the Esophagus, vol. 27, no. 3, pp. 285-293, 2014.

[28] S. Rizvi, S. T. Raza, and F. Mahdi, "Telomere length variations in aging and age-related diseases," Current Aging Science, vol. 7, no. 3, pp. 161-167, 2014.

[29] C. Benoist d'Azy, B. Pereira, F. Chiambaretta, and F. Dutheil, "Oxidative and anti-oxidative stress markers in chronic glaucoma: a systematic review and meta-analysis," PLoS One, vol. 11, no. 12, Article ID e0166915, 2016. 


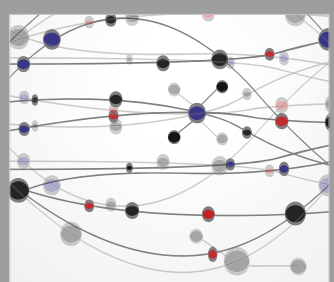

The Scientific World Journal
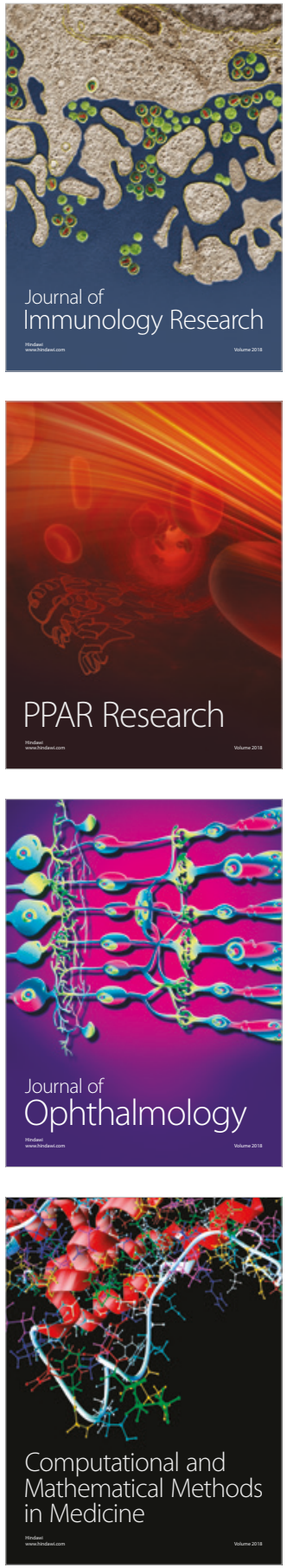

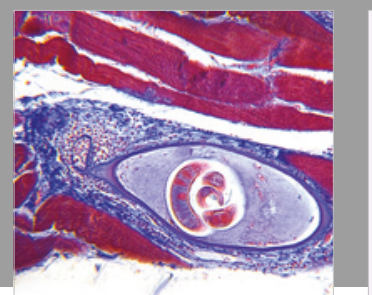

Gastroenterology Research and Practice

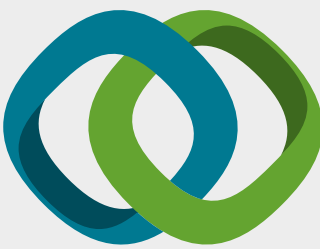

\section{Hindawi}

Submit your manuscripts at

www.hindawi.com
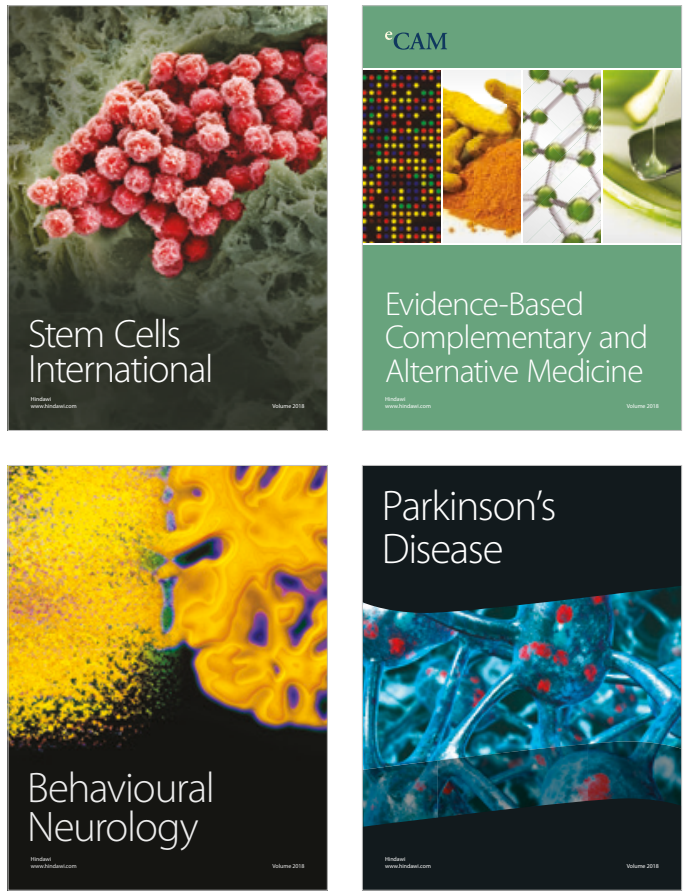

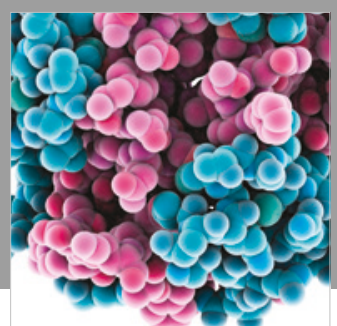

ournal of

Diabetes Research

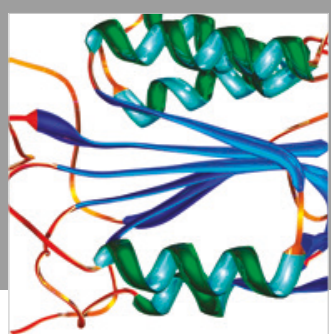

Disease Markers
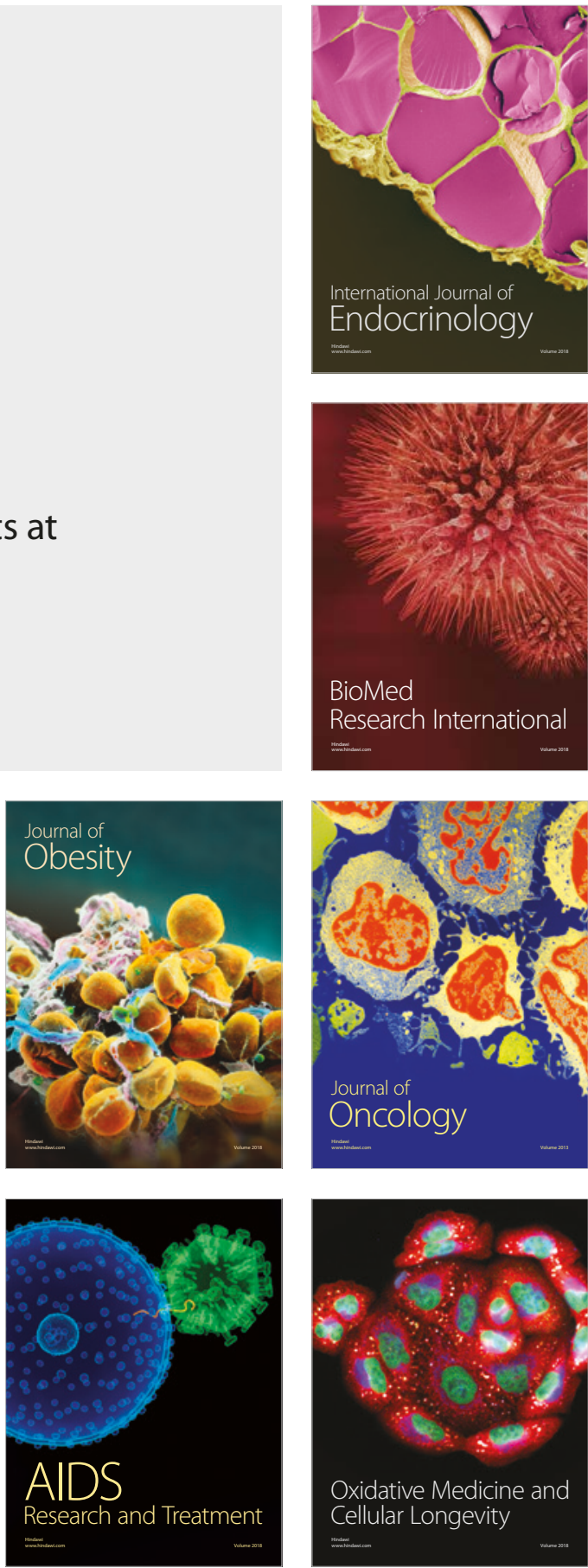